\title{
EDITORIAL
}

\section{Management programmes for heart failure}

\section{A Cheng, $\mathrm{K} \mathrm{Ng}$}

\section{A multidisciplinary team approach is needed for managing heart failure patients}

$\mathrm{H}$ eart failure is a growing epidemic in the world. In the USA, it is estimated that the annual incidence of heart failure is 550000 patients and the number of hospital discharges for heart failure was almost a million in 2001. The cost of treating heart failure in 2004 is estimated to be $\$ 28.8$ billion dollars. Eighty per cent of men under the age of 65 years with heart failure will die within eight years. ${ }^{1}$ Facing these stark statistics, it is recognised that a heart failure disease management programme is necessary to curb the rising cost of managing heart failure and to improve the morbidity and mortality associated with heart failure.

Heart failure disease management (HFDM) recognises that heart failure is a chronic debilitating disease for which the optimal treatment would require a holistic approach adapted to each patient's unique set of medical, psychosocial, behavioural, and financial circumstances. The HFDM includes the following components: heart failure practice guidelines, a multidisciplinary team approach, a target patient population, and a system by which to assess the measures of outcomes of the disease management program.

\section{HEART FAILURE PRACTICE GUIDELINES}

Since 1995, the American Heart Association and the American College of Cardiology have set out guidelines for the pharmacological treatment of heart failure. The latest revision was published in 2001. ${ }^{2}$ Similarly, the European Society of Cardiology and other national cardiac societies have also promulgated guidelines for the management of heart failure. Numerous large multicentre trials have established the evidence base for the usefulness of angiotensin converting enzyme (ACE) inhibitors, second and third generation $\beta$ blockers, angiotensin receptor blockers, digoxin, aldosterone antagonists, and the isosorbide-hydralazine combination in reducing morbidity and mortality in heart failure patients.

See end of article for authors' affiliations

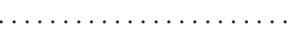

Correspondence to: Dr Alfred Cheng, Mount Elizabeth Medical Centre, 3 Mount Elizabeth, Singapore 228510 alfredcheng18@ hotmail.com

\section{MULTIDISCIPLINARY TEAM APPROACH}

A multidisciplinary team approach is needed in managing heart failure patients because of the numerous barriers to effective heart failure management. The barriers include multiple comorbid medical conditions, polypharmacy, dietary compliance issues, psychosocial concerns, financial constraints, and physical and cognitive limitations. The heart failure management team is usually outpatient based and, in the majority of published studies, revolve around a nurse clinician. Rich and colleagues showed that the multidisciplinary approach reduced readmissions for heart failure in the treatment group $(28.9 \% v$ $42.1 \%)$ as compared to controls. ${ }^{3}$ They also managed to demonstrate a reduction of multiple admissions for heart failure $(6.3 \% v 16.4 \%)$ and an improved quality of life in the treatment group. In a recent systematic review of 11 randomised trials of heart failure disease management programmes, although there was no impact on all cause mortality, there was a significant $23 \%$ reduction in risk of hospitalisation; seven trials demonstrated a significantly shorter length of stay and two out of three trials demonstrated greater use of medications of proven efficacy. ${ }^{4}$ Most importantly, the multidisciplinary model has been shown to reduce the cost of treating heart failure patients. Rich and colleagues demonstrated that with the cost of heart failure intervention factored in, the intervention group still had a cost saving of $\$ 460$ as compared to the control group. ${ }^{3}$

The nurse clinician works closely with the cardiologist for the initiation of medications and the subsequent titration of medications can be done by the nurses following a protocol. The nurse clinician is also involved with patient education, telephonic management, and the counselling of patients. Telephone calls are made to monitor patients for diet compliance, drug compliance, weight control, symptoms, and physical activity. Any problems encountered by the patients would be picked up early and intervened upon utilising the algorithms developed based on clinical guidelines and research. More difficult problems would then be referred to the heart failure clinic where the patient could be assessed by a physician.

Riegel and colleagues reported on telephonic case management using a decision support software program with the nurses calling the patients five days after discharge and up to a total of 14 calls during a six month follow up period in the intervention group. They found a $45.7 \%$ lower hospitalisation rate for heart failure in the intervention group, $43 \%$ less multiple hospitalisations for heart failure, and significantly higher patient satisfaction. ${ }^{5}$ The recently completed DIAL trial in Argentina, which used telephone calls to educate and monitor patients, showed a $20 \%$ reduction in the primary end point of all cause mortality and/or heart failure $(p=0.026)$, a $28 \%$ reduction in admissions for

Abbreviations: $\mathrm{ACE}$, angiotensin converting enzyme; HFDM, heart failure disease management; NYHA, New York Heart Association 
heart failure ( $p=0.005)$, and a number needed to treat of 18 patients to prevent one hospitalisation. ${ }^{6}$ The addition of a clinical pharmacist to the heart failure team was also shown to reduce all cause mortality and non-fatal heart failure events by $78 \%$, and more patients in the intervention group achieved target doses of ACE inhibitors. ${ }^{7}$ The pharmacist was involved in medication evaluation, therapeutic recommendations to the attending physician, patient education, and follow up telemonitoring.

\section{TARGET POPULATION}

HFDM is resource intensive, therefore a population of heart failure patients must be identified in order to be cost effective. Since the main impact of most HFDMs is in the reduction of heart failure readmissions, it would then be more prudent to enrol patients into an HFDM who are considered at "high" risk for heart failure readmission. A prospective study of 257 patients admitted for heart failure identified the following multivariable correlates of readmission or death: single martial status, more comorbid conditions, admission systolic blood pressure of $\leqslant 100 \mathrm{~mm} \mathrm{Hg}$, and the absence of new ST-T wave changes on the initial ECG. ${ }^{8}$ Philbin and DiSalvo used all 1995 discharges in New York State for congestive heart failure involving 42731 patients and found that the predictors of higher risk include black race, Medicare insurance, Medicaid insurance, home health care services after discharge, ischaemic heart disease, valvar heart disease, diabetes mellitus, renal disease, chronic lung disease, idiopathic cardiomyopathy, prior cardiac surgery, and the use of telemetry monitoring during the index hospitalisation. ${ }^{9}$ Krumholz and colleagues identified four risk factors for all cause readmission within six months in patients with a principle discharge diagnosis of heart failure: creatinine $>2.5 \mathrm{mg} / \mathrm{dl}$ $(>220 \mu \mathrm{mol} / \mathrm{l})$ at discharge, prior admission within one year, prior heart failure, and diabetes. ${ }^{10}$ They found that patients with none of the risk factors had a risk of all cause readmission of $26 \%$, whereas patients with three or all the risk factors had an all cause readmission rate of $59 \%$. This method of assessing risk for readmission is by far the easiest to be used in a clinical setting.

\section{MEASURE OF OUTCOMES OF HFDM}

In any disease management programme, there must be a continuous evaluation of the impact of its programme of care. Clinical outcomes measures should include the New York Heart Association (NYHA) functional class, quality of life measures, usage of medications, six minute walk test, unplanned readmission rate, use of emergency services, and compliance to follow up. This evaluation would be important not only as a tool for proof of concept but also to provide checks for a continuous quality improvement programme. Proof of concept is important as not all health care settings are the same, especially because most of the studies are done in developed countries and the same concept might not be fully translatable into the developing countries' health care setting. The addition of patient satisfaction surveys will also help to improve the clinical service in areas in which it is deficient. In 2003, the American Heart Association, the American College of Cardiology, and the Physicians Consortium for Performance Improvement developed a clinical performance measure for heart failure to set standards of care for failure patients during outpatient visits. ${ }^{11}$ The measures include essential laboratory tests, weight, heart rate, blood pressure measurements, assessment of clinical symptoms of volume overload, level of activity, assessment of clinical signs of volume overload, examination of the heart, patient education, and medications used. It is hoped that through these measures the outpatient care for heart failure patients would become standardised and quality improved.

In this issue of Heart, Mejert and colleagues present a study in which heart failure patients randomised to a nurse monitored heart failure programme were compared to those under the care of their community general practitioner. ${ }^{12}$ The study failed to demonstrate any significant difference in mortality, improvement in quality of life, or hospital readmission rate. More patients in the nurse monitored group achieved the goal dose of ACE inhibitor compared to the control group. The absence of any differences between the two groups possibly reflects the already high standard of care provided for heart failure patients by the primary care physicians in Stockholm, who follow a health care plan for heart failure.

\section{FUTURE DIRECTIONS}

Recently, McDonald and colleagues raised the issue of including the in-hospital phase of treatment of heart failure into a disease management programme. ${ }^{13}$ Although their study did not show a reduction in readmissions at one month after in-hospital education, they propose that the in-hospital management of heart failure patients should include care from a heart failure specialist or cardiologist, investigation of the causes of heart failure, clarification of the precipitating factors, and prescription of protocol driven care. They also proposed that the in-hospital phase could be used to educate the patient and the family, and a "two day rule" criteria for assessing readiness for discharge. From our experience, it would be best to incorporate all the above elements into a clinical care path which clinicians can follow during their care of the patients.

Secondly, another aspect often overlooked is the incorporation of cardiac rehabilitation into HFDM. In a small study of 99 patients in NYHA class II-IV randomised to 14 months of exercise training or control, a stunning $71 \%$ reduction in hospital readmissions for heart failure and $63 \%$ reduction in cardiac deaths was found. ${ }^{14}$ If these results are borne out by larger studies that are ongoing, it would become an important pillar in an HFDM programme.

Lastly, as heart failure is a chronic disease process in patients, there would come a time whereby discharge from a HFDM programme would be anticipated, either when the patient dies or receives a transplant, or is stable enough to be discharged to the community primary care physician. There are currently no studies looking at the long term outcome of such patients and how primary care physicians can continue the care and education of heart failure patients and how they can interface with the hospital based HFDM programmes.

\section{Authors' affiliations \\ A Cheng, Mount Elizabeth Hospital, Singapore \\ A Cheng, K Ng, Tan Tock Seng Hospital, Singapore}

\section{REFERENCES}

1 American Heart Assocation. American Heart Association heart disease and stroke statistics-2004 update. Dallas, Texas: AHA, 2003.

2 Hunt S, Baker DW, Chin MH, et al. ACC/AHA guidelines for the evaluation and management of chronic heart failure in the adult: executive summary. A report of the American College of Cardiology/American Heart Association task force on practice guidelines (committee to revise the 1995 guidelines for the evaluation and management of heart failure). Circulation 2001;104:2996-3007.

3 Rich MW, Beckham V, Wittenberg C, et al. A multidisciplinary intervention to prevent the readmission of elderly patients with congestive heart failure. N Engl J Med 1995;333:1190-5.

4 McAlister FA, Lawson FME, Teo KK, et al. A systematic review of randomized trials of disease management programs in heart failure. Am J Med $2001 ; 110: 378-84$.

5 Riegel B, Carlson B, Kopp Z, et al. Effect of a standardized nurse casemanagement telephone intervention on resource use in patients with chronic heart failure. Arch Intern Med 2002;162:705-12. 
6 Grancelli H, Varini S, Ferrante D, for the GESICA Investigators, et al. Randomized trial of telephone intervention in chronic heart failure (DIAL): study design and preliminary observations. J Card Fail 2003;9:172-9.

7 Gattis WA, Hasselblad V. Whellan DJ, et al. Reduction in heart failure events by the addition of a clinical pharmacist to the heart failure management team. Arch Intern Med 1999;159:1939-45.

8 Chin MH, Goldman L. Correlates of early hospital readmission or death in patients with congestive heart failure. Am J Cardiol 1997;79:1640-4

9 Philbin EF, DiSalvo TG. Prediction of hospital readmission for heart failure: development of a simple risk score based on administrative data. J Am Coll Cardiol 1999;33:1560-6.
10 Krumholz HM, Chen YT, Wang Y, et al. Predictors of readmission among elderly survivors of admission with heart failure. Am Heart J 2000;139:72-7.

11 American Heart Association. www.americanheart.org/downloadable/ heart/105579825669HFMMiniSetR030157 final.pdf

12 Meihert $M$, Kahan $\mathrm{T}$, Persson $\mathrm{H}$, et al. Limited long term effects of a management programme for heart failure. Heart 2004;90:1010-15.

13 McDonald K, Ledwidge M. Heart failure management programs: can we afford to ignore the inpatient phase of care? J Card Fail 2003;9:258-62.

14 Belardinelli R, Georgiou D, Cianci G, et al. Randomized, controlled trial of long term moderate exercise training in chronic heart failure: effects on functional capacity, quality of life, and clinical outcome. Circulation 1999;99:1173-82.

\section{IMAGES IN CARDIOLOGY}

\section{Safe biopsy of aortic masses guided by intraluminal two dimensional ultrasonography}

$\mathrm{U}$

sing transoesophageal echocardio-

graphy, large aortic masses were

found to be rigidly attached to the aortic wall in two patients-in one patient the masses extended from the aortic arch to the descending aorta, while in the other patient the masses extended from the descending thoracic to the abdominal aorta. Intraluminal two dimensional ultrasound was then used to guide percutaneous biopsies of these aortic masses. First, a long 11 French access sheath was conventionally introduced into the left femoral artery and advanced to the descending aorta. A phased array transducer tipped AcuNav-Catheter (Siemens-Acuson Inc) was then inserted through the access sheath so that only the tip of the catheter with the transducer was allowed to leave the sheath. Next, the access sheath with the AcuNav-Catheter inside was slightly withdrawn and the transducer adjusted to the distal end of the mass by observing the ultrasonographic images (left panel). Under continuous ultrasonographic viewing, each three targeted biopsies were taken from the depth of the masses (right upper panel) using a radial-jaw-biopsy-forceps (Boston Scientific Inc), previously inserted via an 8 French multi-purpose guiding catheter which was introduced through a second access sheath from the right femoral artery. Opening, pushing, and closure of the biopsy forceps were precisely guided and documented (right lower panel). No complications were observed. In addition to fresh fibrin and erythrocytes, histological analyses revealed connective tissue with fibroblasts and macrophages in all six specimens.

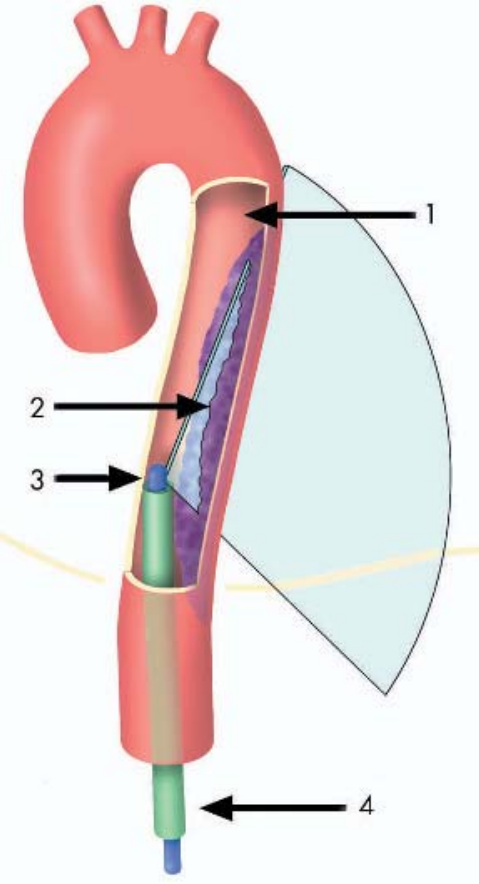

Schematic drawing of the intra-aortic sonographic approach to an intraluminal mass. 1, aorta; 2, intra-aortic mass; 3, transducer tipped AcuNav-Catheter with its tip allowed to leave the sheath; 4, long sheath previously advanced through the femoral artery.

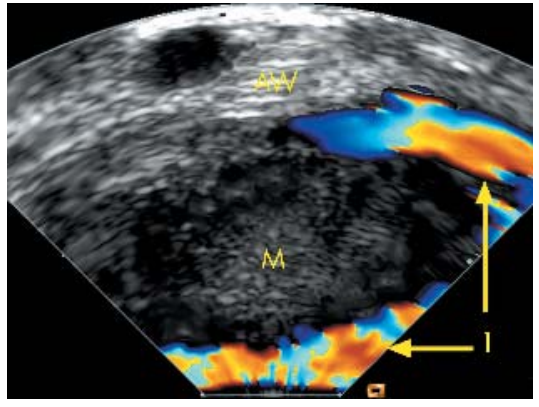

Intra-aortic mass $(M)$ rigidly attached to the aortic wall (AW) and blood flow. 1, blood flow surrounding the mass.

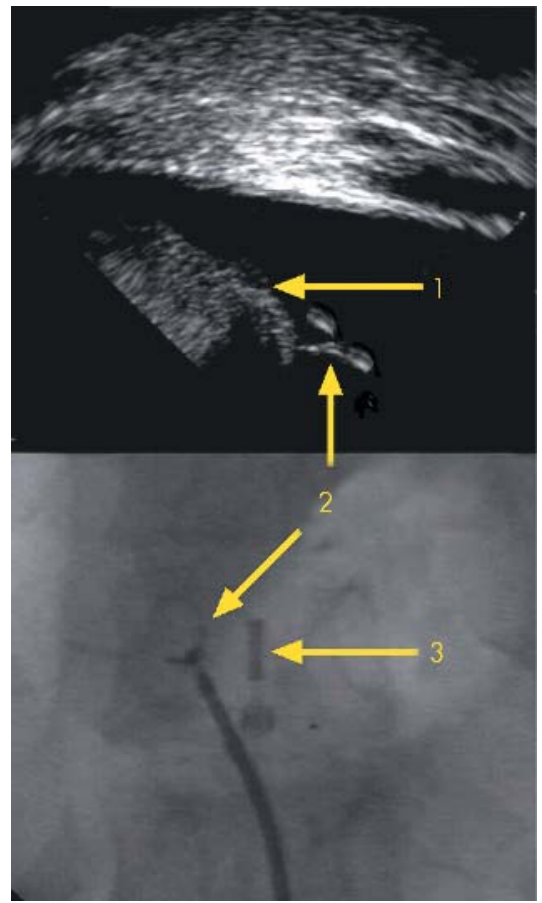

Opened biopsy forceps approaching the mass for biopsy retrieval. 1, mass; 2, opened biopsy forceps; 3, transducer tipped AcuNav-Catheter. 